\title{
MEDIDOR DIDÁTICO DA QUALIDADE DO LEITE PRODUZIDO PELO PEQUENO PRODUTOR RURAL
}

Wesley Matheus Araújo da Silva -wmas@discente.ifpe.edu.br

Vitoria Borges Santana-vbs@discente.ifpe.edu.br

Manoel Alves Cordeiro Neto - manoel.neto@garanhuns.ifpe.edu.br

Gerônimo Barbosa Alexandre - geronimo.alexandre@garanhuns.ifpe.edu.br

Instituto Federal de Educação Ciência e Tecnologia de Pernambuco

R. Padre Agobar Valença, Severiano Moraes Filho

55299-390- Garanhuns - Pernambuco

Resumo: $O$ presente trabalho consiste no projeto e na montagem de uma sonda de medição de parâmetros físicos, químicos e elétricos na refrigeração do leite. O sistema conta com sistema de medição, armazenamento de dados e sistema de monitoramento remoto e visa dar suporte ao pequeno produtor rural detalhando em tempo real parâmetros como o pH e temperatura do leite assim como a curva de demanda elétrica consumida pelos equipamentos, por meio de hardware de baixo custo. O diferencial do trabalho está em: (1) supervisão remota de dados; (2) baixo custo; (3) baixo consumo de energia pelos componentes utilizados no protótipo; (4) caráter didático; (5) medição de maior número de variáveis.

Palavras-chave: Protótipo. Project-based learning. Instrumentação eletrônica.

\section{INTRODUÇÃO}

A indústria de laticínios é um importante ramo da agropecuária brasileira. O leite representa $24 \%$ do valor bruto da produção (VBP) gerado pela pecuária (ZOCCAL, 2017). Para que o leite possa ser utilizado pela indústria o produtor deve tomar uma série de medidas imposta pela EMBRAPA (Empresa Brasileira de Pesquisa Agropecuária).

Uma dessas medidas diz que o leite deve ter sua temperatura reduzida para $4^{\circ} \mathrm{C}$ em até duas horas após a ordenha, e para realizar essa tarefa os produtores utilizam tanques de refrigeramento, que por sua vez nada mais são que grandes geladeiras que ligam e desligam de acordo com a temperatura do leite armazenado. Através desses tanques a quantidade de leite desclassificado por acidez diminuiu bastante visto que antes da popularização desses tanques o leite era armazenado em latões de até 50 litros, muitas vezes expostos a altas temperaturas que aumentavam a acidez do leite e diminuindo assim sua qualidade. Mesmo assim, indubitavelmente ainda há muito a ser melhorado no tocante à produção do leite de forma geral.

Sendo assim, a motivação deste projeto surgiu da necessidade do desenvolvimento de ferramentas inteligentes para a melhoria do leite produzido e ofertado por pequenos produtores rurais do Agreste Meridional Pernambucano, em especial na fase de estocagem e refrigeração do leite. A região de atuação do trabalho é a segunda maior bacia leiteira do estado do Pernambuco, uma área geográfica de abrangência do semiárido brasileiro, com clima quente e que clama pela introdução de novas tecnologias para o aperfeiçoamento da atividade produtivas locais. 
Outra motivação para o projeto foi a necessidade de atividades envolvendo aplicações práticas no curso de Engenharia Elétrica do Instituto Federal de Pernambuco campus Garanhuns, especialmente na disciplina de Microcontroladores. A metodologia de ensino baseada em projetos (Project-based learning) propõe a atividade prática como ferramenta de aprendizagem e aproxima o aluno de aplicações das disciplinas no mercado de trabalho.

As ferramentas desenvolvidas são uma sonda de medição de parâmetros com supervisão de dados em tempo real e um medidor de energia que informa o consumo diário do sistema de refrigeração.

Outro diferencial é o fato de não ainda não existir no mercado uma tecnologia que agrega todos os medidores em um único equipamento, além do desconhecimento dos produtores sobre tais tecnologias, muito menos no atendimento as prescrições legais da qualidade do leite. O desenvolvimento e a implantação de novas tecnologias na refrigeração do leite trazem ganhos econômicos e garantem a qualidade no produto comercializado.

Entre os benefícios para o produtor rural, temos: redução na fatura de energia, monitoramento da qualidade do leite, prevenção de desperdícios, atendimento de prescrições legais para venda e armazenamento do leite e maior controle do consumo de energia mensal.

\section{METODOLOGIA DE ENSINO BASEADA EM PROJETOS}

Por se tratar de uma área inerentemente multidisciplinar, a engenharia de automação e controle envolve conceitos e problemas de diversas áreas. Neste contexto o ensino baseado em projetos deve ser iniciado nos primeiros anos do curso para que os alunos possam se antecipar as vivencias industriais.

$\mathrm{Na}$ engenharia elétrica, o desenvolvimento de protótipos de medidores didático pode ser introduzido em diversas disciplinas tais como: Instrumentação Industrial, Microcontroladores, Eletrônica, Software Supervisório, Redes Industrias. Estas últimas de maneira específica dentro da disciplina, já na disciplina Projeto Integrador há a concepção de produtos por meio da integração de várias disciplinas.

Nesta seção, aborda-se o uso do medidor didático inteligente da qualidade do leite como uma ferramenta de ensino no contexto da disciplina Microcontroladores, a qual se trata de uma disciplina obrigatória oferecida para alunos a partir do $6^{\circ}$ Semestre do curso de graduação em Engenharia Elétrica do Instituto Federal de Pernambuco Campus Garanhuns.

\subsection{Metodologia Empregada na Disciplina}

No ensino baseado em projetos o aluno passa ser agente ativo do processo e o professor atua como um consultor, orientando o aluno nas etapas propostas, auxiliando na melhor solução e no feedback de informações técnicas, na gestão de tempo e na gestão dos materiais.

No início do semestre, os alunos são apresentados aos temas propostos para o projeto e a montagem dos medidores didáticos, sendo estimulados a discutirem sobre o funcionamento de cada medidor. Em seguida a Turma é dividida em grupos e por meio de sorteio, o medidor didático a ser confeccionado é escolhido.

A partir das aulas teóricas sobre os diversos tipos de medidores usados na aferição de grandezas físicas industriais é solicitado aos grupos o projeto do medidor escolhido (desenhos 2D e 3D, lista de materiais, orçamento e diagramas elétricos).

Ainda na Unidade I são solicitados os testes individuais de cada componente usado e a montagem física do protótipo. Já na Unidade II são solicitados os testes do medidor didático construído e a validação da supervisão local e remota dos dados medidos. 
Há semestres letivos onde poderá haver apenas uma proposta de medidor didático, como há semestres letivos com várias propostas de medidores. No Semestre letivo 2019.2 foi proposto a construção e a validação de quatro protótipos diferentes: (1) Medidor da qualidade do leite; (2) Medidor da qualidade do ar; (3) medidor da qualidade da água; (4) medidor integrado de volume, pressão e umidade em silos.

A turma foi dividida em 04 (quatro) grupos que confeccionaram quatro protótipos, dois grupos optaram pelo uso da tecnologia Bluetooth para supervisão dos dados, um grupo optou pelo uso da plataforma de internet das coisas (ThingSpeak), um grupo optou pela criação de um servidor Web.

Neste Trabalho será apresentado o medidor inteligente de variáveis elétricas, químicas e físicas na refrigeração do leite estocado em cooperativas (Projeto 1) com supervisão de dados pela plataforma ThingSpeak desenvolvido pelo Grupo 3.

\section{MATERIAIS E MÉTODOS}

A presente pesquisa caracteriza-se como teórico-experimental. Inicialmente, na fase teórica, realizou-se a revisão bibliográfica por meio de livros, artigos e sites acadêmicos e especializados para compreensão do problema e paralelamente foram feitas consultas de protótipos já desenvolvidos (academicamente) e produtos já comercializados, a nível nacional e internacional. Por meio desta pesquisa foi possível avaliar erros e acertos em projetos semelhantes e dessa forma modificar alguns detalhes no trabalho. Além disso, foi iniciado o desenvolvimento do código em $\mathrm{C}$ a ser utilizado no protótipo.

Em seguida, foram feitas simulações do protótipo e do código em softwares específicos, como o Fritzing, Quickcad, Proteus e a própria plataforma do microcontrolador utilizado. Após essa etapa, foi elaborada uma lista de materiais a serem utilizados na confecção do protótipo experimental.

Os materiais utilizados para confecção do protótipo experimental estão dispostos na Tabela 1. O custo total do protótipo foi baixo ( $\mathrm{R} \$ 336,00)$ quando comparado a uma sonda de medição comercial desenvolvida pela empresa cearense RAV Tecnologia $(\mathrm{R} \$ 800,00)$.

Tabela 1 - Orçamento do projeto.

\begin{tabular}{cc}
\hline Material & Preço (médio) \\
\hline Fonte 5V & $\mathrm{R} \$ 15,90$ \\
\hline Display LCD & $\mathrm{R} \$ 27,90$ \\
\hline Protoboard para montagem & $\mathrm{R} \$ 11,60$ \\
\hline ESP32 & $\mathrm{R} \$ 60,00$ \\
\hline Sensor de pressão & $\mathrm{R} \$ 75,00$ \\
\hline Sensor de pH & $\mathrm{R} \$ 64,13$ \\
\hline Sensor ultrassônico & $\mathrm{R} \$ 17,22$ \\
\hline Sensor de temperatura & $\mathrm{R} \$ 15,21$ \\
\hline Sensor de corrente & $\mathrm{R} \$ 48,90$ \\
\hline TOTAL & $\mathrm{R} \$ 335,86$ \\
\hline Fonte: Autores (2020).
\end{tabular}

Inicialmente, o microcontrolador escolhido foi a placa Arduino Uno R3, porém o mesmo não atendeu as necessidades do projeto devido ao seu número limitado de portas. Dessa forma, escolheu-se a placa Arduino MEGA 2560 devido ao seu amplo número de saídas e entradas digitais. Porém esse microcontrolador ainda apresentou limitações como a falta da conexão Bluetooth e Wi-Fi em um único hardware. 
Sendo assim, foi escolhido o ESP32/Espressif Devkit ESP-WROOM-32 V1.0 como o microcontrolador do projeto, pois além de possuir uma grande quantidade de portas disponíveis, permite a conexão sem fio. Este microcontrolador se mostrou uma ferramenta muito útil para solucionar o problema que motivou a criação do projeto, já que além de ter um custo baixo e a capacidade de se comunicar de forma wireless, também pode ser programado através da IDE Arduino, que é open source, tornando possível a customização do projeto.

Com os materiais em mãos, foi realizada a montagem do protótipo, que por sua vez foi submetido a testes e assim sofreu algumas alterações, assim como o código montado anteriormente.

O medidor experimental foi desenvolvido no Laboratório de Eletrônica e Circuitos Elétricos, do Instituto Federal de Educação, Ciência e Tecnologia de Pernambuco campus Garanhuns. O protótipo final foi calibrado e validado inicialmente em laboratório, depois foi utilizado em oficina experimental realizada pelos extensionistas e professores do IFPE nas comunidades rurais.

Após este trabalho de calibração do medidor e divulgação do produto, houve a instalação e os testes de aceitação em um tanque de 1.000 litros cedido pelas empresas parceiras do projeto: a Associação dos Moradores e Produtores Rurais de Campo Grande e Região, a Fazenda Marcos Rogério Rocha Malta e o Laticínio Belo (Povoado Campo Grande / Águas Belas - PE).

Dentre os sensores utilizados podemos destacar:

- O sensor de temperatura DS18B20 a prova d'água do tipo sonda é um dos componentes mais utilizados em projetos que envolva medição de temperatura em ambientes úmidos ou em recipientes com liquido. $\mathrm{O}$ sensor é revestido por um material à prova d'água e sua ponta é encapsulada em aço inoxidável. (OLIVEIRA, 2019).

- O JSN-SR04T é um sensor de distância ultrassônico à prova d'água, fácil de usar, com uma faixa de 25 a $450 \mathrm{~cm}$ (FELIPE,2019).

- O Sensor de $\mathrm{pH}$ Arduino modelo PH-4502C é um sensor muito funcional e prático desenvolvido especialmente para trabalhar em conjunto com microcontroladores, podendo atuar em inclusive com o Raspberry $\mathrm{Pi}$. De forma simplificada, $\mathrm{pH}$ significa potencial hidrogeniônico (quantidade de prótons $\mathrm{H}+$ ), possibilitando indicar a neutralidade, acidez ou mesmo a alcalinidade de uma solução líquida. (USINAINFO).

- O display Nokia, este possui ótima relação custo $\mathrm{x}$ benefício para o seu projeto, pois se trata de um display gráfico monocromático com resolução de $84 \times 48$ pixels, e com ele você pode ter em uma mesma tela gráficos e texto. (FELIPEFLOP).

Foi realizado também um estudo para escolher o formato e a localização (em relação ao tanque onde será instalada) mais adequados possíveis para a sonda. Nessa etapa foi realizada uma comparação entre o medidor de energia montado e o modelo comercializado, o AEOTEC Energy Meter ( $\mathrm{R} \$ 350,00)$, sendo avaliado o envio / recebimento dos dados medidos e a calibração do sensor de corrente.

Na Figura 1 é ilustrado o design 3D do medidor inteligente proposto instalado no tanque de refrigeração. O medidor de energia estará conectado ao cabo de alimentação do tanque.

Figura 1 - Detalhes da instalação da sonda de medição no tanque de refrigeração do leite. 


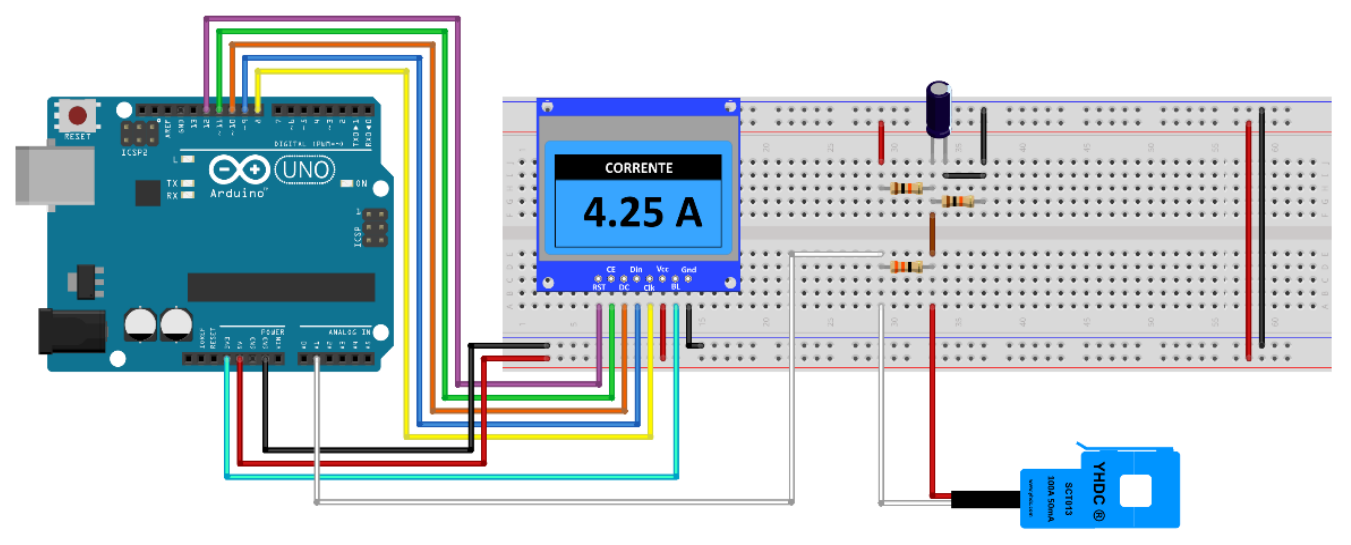

Fonte: Autores (2020).

Figura 4 - Diagrama elétrico do medidor de variáveis físico-químicas do leite.

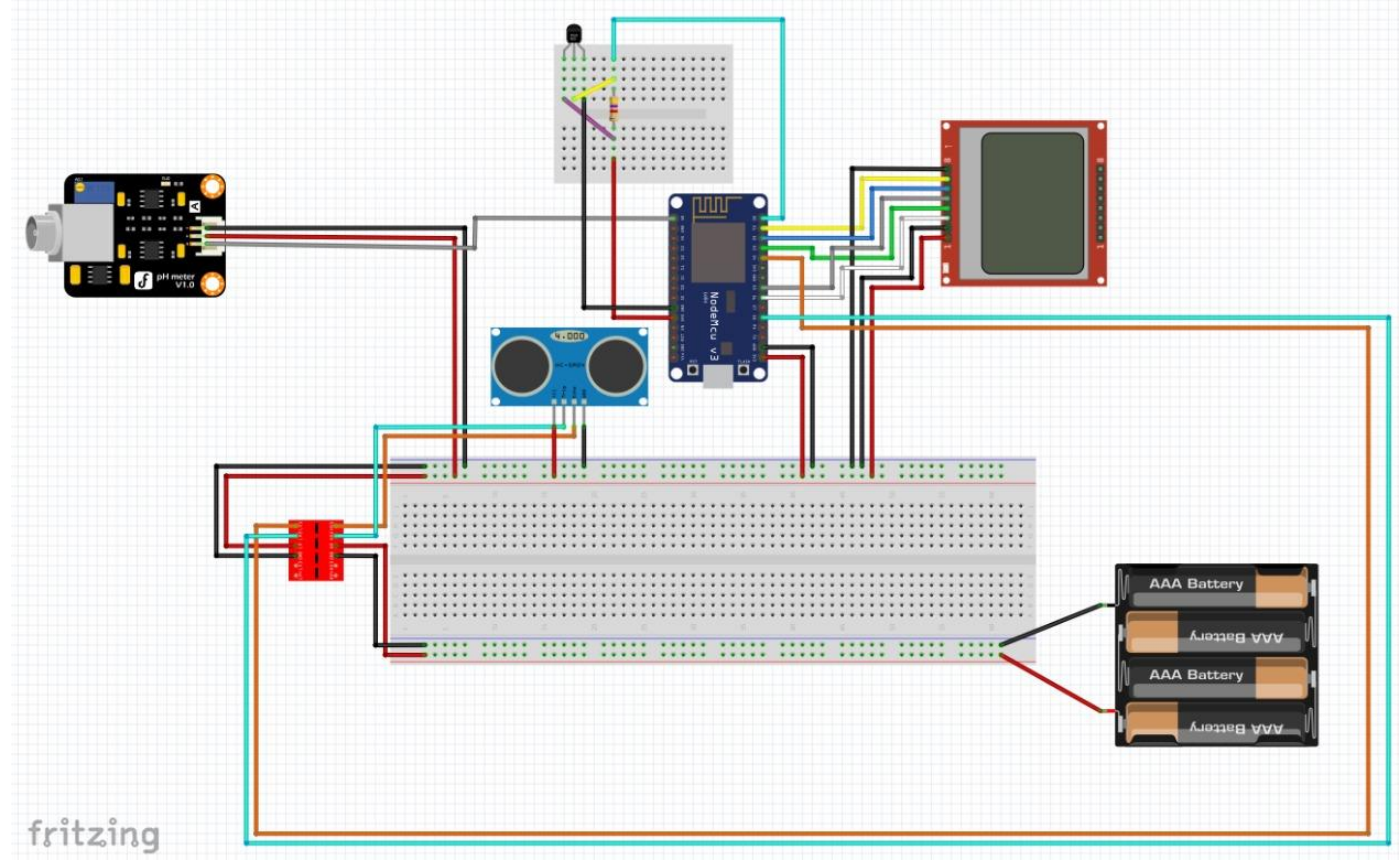

Na Figura 3 é ilustrado o esquemático da ligação do sensor de corrente ao microcontrolador, já na Figura 4 é ilustrado o diagrama elétrico de montagem do medidor de variáveis físico-químicos. Os dois juntos formam a sonda de medição inteligente da qualidade do leite refrigerado instalado no tanque de armazenamento de leite da cooperativa dos Moradores e Produtores Rurais de Campo Grande e Região localizada no município de Águas Belas-PE.

\section{RESULTADOS E DISCUSSÃO}

Em relação ao medidor de energia, o sensor de corrente utilizado possui características técnicas que atenderam bem o produto, principalmente quando analisada a questão custobenefício; porém não é de performance idêntica à um sensor profissional de alto desempenho e pode apresentar desvios nas medições. Para amenizar essa dificuldade, a comparação dos dados obtidos pela estação de baixo custo com dados obtidos pelo sensor de performance 
profissional, como o próprio AEOTEC Energy Meter, pode ajudar a calibrar o medidor proposto aumentando o desempenho ao longo do tempo.

Na Figura 5 é ilustrado o protótipo montado em protoboard para os testes de calibração dos sensores de temperatura, $\mathrm{pH}$, volume do tanque e energia ativa consumida pelo equipamento de refrigeração. Na Figura 6 é ilustrado o resultado das medições de calibração dos sensores em laboratório.

$\mathrm{Na}$ Figura 6(a) é ilustrado a medição de $21,5^{\circ} \mathrm{C}$ pelo sensor DS18B20 do líquido contido no tanque, já o volume do tanque é de $2.014 \mathrm{~L}$, sendo aferido de modo indireto pela leitura da distância feita pelo sensor ultrassônico e na programação convertido em volume.

$\mathrm{Na}$ Figura 6(b) é ilustrado o valor de corrente elétrica mensurada pelo sensor de efeito hall modelo SCT 013-000 100 A, quando o sensor foi conectado a um cabo elétrico em um circuito de corrente alternada de baixa tensão.

Figura 5 - (a) Medidor das variáveis físico-químicas. (b) Medidor das variáveis elétricas.

(a)

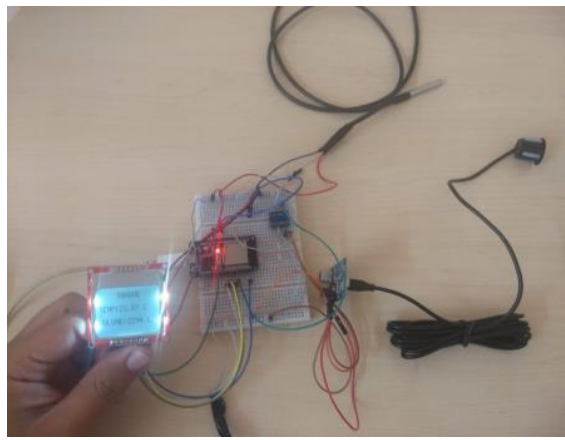

(b)

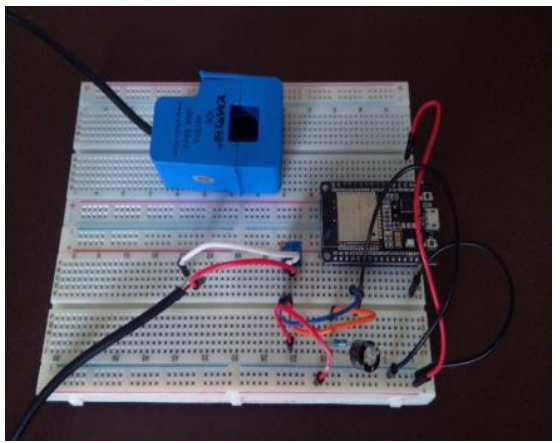

Figura 6 - (a) Medições físico-químicas. (b) Medições de grandezas elétricas.

(a)

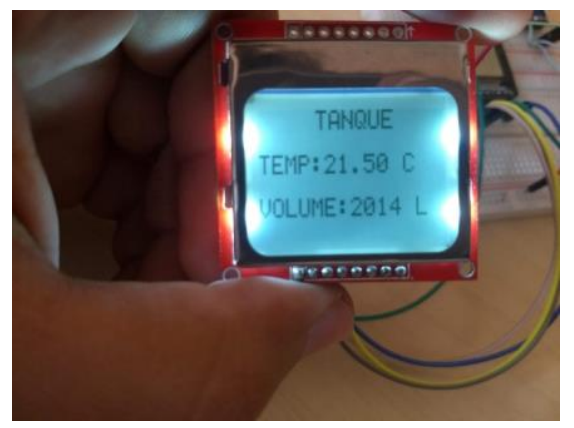

(b)

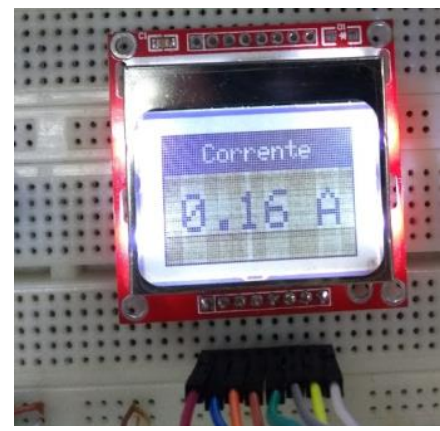

O sensor de corrente é o "coração" do medidor de energia, pois ele é responsável pela medição indireta da potência elétrica $\left(\mathrm{P}=220^{*} \mathrm{I}\right)$, esta quando multiplicada pelo tempo tem-se a energia elétrica consumida por hora $\left(E=\mathrm{P}^{*} \Delta \mathrm{t}\right)$.

A Figura 7 ilustra uma visão geral do canal confeccionado usando a plataforma ThingSpeak, que recebe e disponibiliza os dados enviados pelo protótipo desenvolvido. Esses gráficos são gerados da seguinte maneira: os sensores enviam via sinal digital ou analógico os dados obtidos para o microcontrolador que faz o processamento desses dados de acordo com o código fonte, em seguida envia via MQTT os dados processados para o canal confeccionado no ThingSpeak. 
O ThingSpeak possui apenas uma limitação: o tempo entre upload de dados deve ser de, no mínimo, 15 segundos. Se isso for desobedecido, os dados enviados fora deste intervalo de tempo serão ignorados ou não registrados. No experimento realizado os uploads foram feitos em intervalos de 60 segundos.

O fluxograma de dados na nuvem usando o protocolo MQTT é ilustrado na Figura 8, O Publisher do projeto será o microcontrolador ESP32 que enviará os dados de todos os sensores, a plataforma ThingSpeak desempenhará o papel de Broker e qualquer pessoa que acessar o canal disponibilizado pelo sistema será o Subscriber.

Figura 7 - Tela de monitoramento via internet.

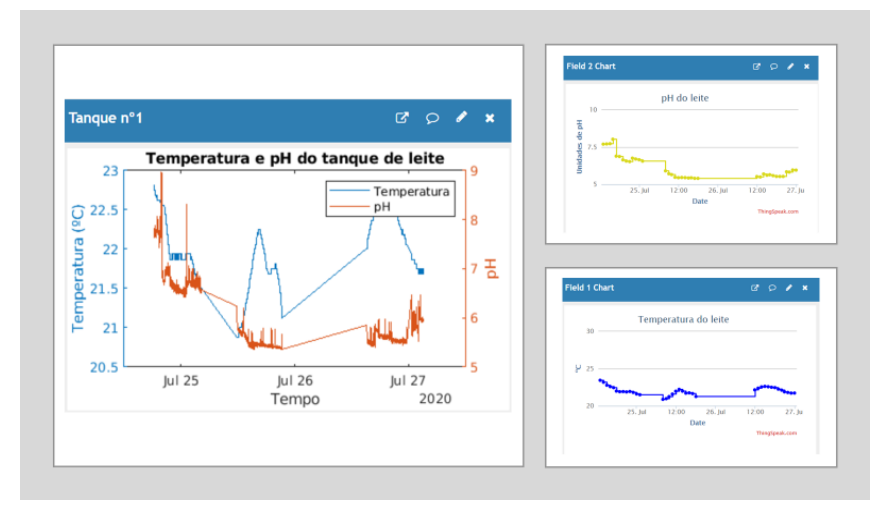

Fonte: Autores (2020).

Figura 8 - Fluxo de dados na supervisão remota.

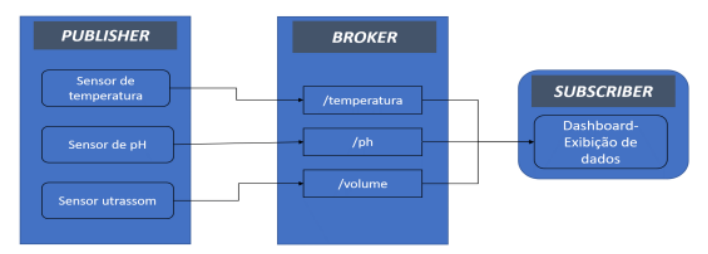

Fonte: Autores (2020).

Na versão gratuita da ferramenta ThingSpeak há limitações de nove gráficos simultâneos por canal, sendo oito para as medições e o nono gráfico diz respeito ao ponto de localização do medidor (coordenadas de latitude, longitude e elevação). No canal criado existe uma seção chamada "metadata" onde é possível inserir informações que utilizem os dados medidos para gerar arquivos do tipo JSON, XML, CSV. A ferramenta permite ao administrador a inserção de um único vídeo do YouTube (por exemplo, o link no YouTube para apresentação do canal confeccionado) e a exportação do código fonte para a plataforma Github (nuvem de compartilhamento de códigos abertos na Web).

Todos os dados medidos na bancada didática (leituras dos sensores) são condicionados na plataforma Arduino e enviados para um banco de dados no MS Excel para fins de registro operacional do processo. Além do armazenamento dos dados, foi utilizado a ferramenta PLXDAQ para plotagem em tempo real dos dados enviados pelo microcontrolador ao MS Excel.

As principais dificuldades encontradas foram: leituras de diagramas elétricos, programação em C para o microcontrolador, dinâmica lenta da eletroválvula e conhecimentos em montagens de circuitos eletroeletrônicos. 
Uma informação importante observada na maioria dos alunos após o termino da Disciplina foi a motivação da turma em estudar/conhecer a disciplina, quando esta foi assistida por atividades práticas. A metodologia convencional (aula expositiva e provas escritas) torna a tarefa de ensinar cansativa e de difícil compreensão devido ao quantitativo de equações e cálculos envolvidos, bem como o nível de abstração por parte dos alunos.

Outro resultado positivo diz respeito ao quantitativo de discentes que enxergaram aplicações reais da disciplina, um dos desafios da realidade do futuro engenheiro. A metodologia de ensino baseada em projetos propicia uma aprendizagem inserida no contexto educacional atual, rompendo com a forma rígida e pré-estabelecida de transmissão dos conteúdos. O envolvimento e o desempenho dos alunos na disciplina de Microncontroladores utilizando essa metodologia foram visivelmente melhores quando comparado à metologia tradicional de ensino devido à liberdade de trabalhar os conteúdos da disciplina em seu próprio ritmo. A visualização de aplicações práticas dos conteúdos trabalhados em sala de aula (ao invés da confecção de cálculos abstratos e simulações) também ajudou os alunos a relacionar conteúdos interdisciplinares de forma integrada, promovendo uma melhor absorção dos conteúdos da disciplina.

\section{CONSIDERAÇÕES FINAIS}

O trabalho de ensino baseado em montagens favoreceu a imersão dos estudantes do curso de engenharia elétrica que vivenciaram a dinâmica, nas disciplinas de eletrônica, programação, mecânica e sistemas embarcados.

As experiências adquiridas tais como: trabalho em equipe e com segurança atentando para as prescrições legais, leitura de manuais, elaboração de projetos, a experiência ao falar em público, manuseio das ferramentas básicas de manutenção elétrica, aprendizagem no uso de manuais de equipamentos elétricos, aprendizagem em organização e guarda de materiais elétricos. Na visão dos alunos é uma antecipação do trabalho do futuro engenheiro nas linhas montagens em fábricas.

O protótipo desenvolvido apresentou baixo custo quando comparado com produto similar comercial, dados confiáveis e precisão nas medições; sendo uma ferramenta didática de apoio ao ensino-aprendizagem no ensino da graduação em engenharia elétrica. O diferencial dos produtos está na supervisão remota dos dados medidos e na disponibilidade das medições para qualquer usuário conectado à internet, seja por computadores ou dispositivos móveis.

Em relação à perspectiva de trabalhos futuros tem-se: (a) projetar e incorporar maior número de sensores; (b) projetar e incorporar um sistema especialista para detecção e diagnóstico de falhas nos sensores, nos atuadores e no tanque; (c) explorar a capacidade do ESP32 na criação de um servidor Web; (d) desenvolvimento de aplicativo Bluetooth / Web para monitoramento via dispositivos móveis.

\section{Agradecimentos}

Ao Instituto Federal do Pernambuco Campus Garanhuns.

\section{REFERÊNCIAS}

TIPÁN, L. F e RUMIPAMBA, J.A. Medidor inteligente de energía eléctrica utilizando la tarjeta electrónica Raspberry Pi. Revista Técnica Energía. Edición nº 14, Enero 2018. ISSN $1390-5074$. 
"Os desafios para formar hoje o engenheiro do amanhã"

ZOCCAL, R. A atividade leiteira retratada no Censo Agropecuário 2017. Revista Balde Branco. Vol. 53 n. 646. São Paulo - SP: Balde Branco. P. 16-17. 2018.

DULES, L. F., et al. Desenvolvimento de um sistema móvel e automático a telemetria para monitoramento de temperaturas em águas poluídas de rios e lagoas. Anais, VII Congresso Norte e Nordeste de Pesquisa e Inovação (CONNEPI), Palmas - TO, 2012.

SANTANA, T. S., et al. Monitoramento em tempo real de parâmetros físico-químicos: pH e temperatura, usando hardware livre. Anais, VI Congresso de Iniciação científica do IF Goiano, Goiânia-GO, 2017.

FELIPEFLOP. Display LCD Nokia 5110. Disponível em: https://www.filipeflop.com/produto/display-lcd-nokia-5110/. Acesso em: 29 de maio de 2020.

FELIPE, N. Sensor ultrassônico JSN. Disponível em: https://niltonfelipe.wordpress.com/2019/05/29/sensor-ultrasonico-jsn-sr04t/. Acesso em: 29 de maio de 2020.

OLIVEIRA, E. Como usar com Arduino - Sensor de Temperatura DS18B20 Prova D'água do Tipo Sonda. Disponível em: https://blogmasterwalkershop.com.br/arduino/comousar-com-arduino-sensor-de-temperatura-ds18b20-prova-dagua-do-tipo-sonda/. Acesso em: 29 de maio de 2020.

SULINOX, Ordenhadeiras. Manual do proprietário: Tanque refrigerador de leite a granel. Disponível em: http://sulinox.com/manuais/Resfriador_de_Leite.pdf. Acesso em: 24 maio de 2020.

USINAINFO. Sensor de pH Arduino + Módulo de Leitura. Disponível em: https://www.usinainfo.com.br/outros-sensores-arduino/sensor-de-ph-arduino-modulo-deleitura-5316.html. Acesso em: 29 de maio de 2020.

\section{DIDACTIC MILK QUALITY METER PRODUCED BY THE SMALL RURAL PRODUCER}

Abstract: This study aimed at the design and assembly of a probe to measure physical, chemical and electrical parameters in milk refrigeration. The system relies on the measurement system, data storage and remote monitoring system and aims to support small farmers. Detailing in real time parameters such as milk $\mathrm{pH}$ and temperature as well as the electrical demand curve consumed by the equipment, using low cost hardware. The work differential is in: (1) remote data supervision; (2) low cost; (3) low energy consumption by the components used in the prototype; (4) didactic character; (5) measurement of a greater number of variables.

Keywords: Prototype, Project based-learning, Electronic instrumentation. 